PROCEEDINGS OF THE

AMERICAN MATHEMATICAL SOCIETY

Volume 130, Number 2, Pages 489-505

S 0002-9939(01)06132-9

Article electronically published on June 11, 2001

\title{
AN IMPROVED HARDY-SOBOLEV INEQUALITY AND ITS APPLICATION
}

\author{
ADIMURTHI, NIRMALENDU CHAUDHURI, AND MYTHILY RAMASWAMY
}

(Communicated by David S. Tartakoff)

\begin{abstract}
For $\Omega \subset \mathbb{R}^{n}, n \geq 2$, a bounded domain, and for $1<p<n$, we improve the Hardy-Sobolev inequality by adding a term with a singular weight of the type $\left(\frac{1}{\log (1 /|x|)}\right)^{2}$. We show that this weight function is optimal in the sense that the inequality fails for any other weight function more singular than this one. Moreover, we show that a series of finite terms can be added to improve the Hardy-Sobolev inequality, which answers a question of BrezisVazquez. Finally, we use this result to analyze the behaviour of the first eigenvalue of the operator $L_{\mu} u:=-\left(\operatorname{div}\left(|\nabla u|^{p-2} \nabla u\right)+\frac{\mu}{|x|^{p}}|u|^{p-2} u\right)$ as $\mu$ increases to $\left(\frac{n-p}{p}\right)^{p}$ for $1<p<n$.
\end{abstract}

\section{InTRODUCTION AND RESUlts}

Let $\Omega$ be a bounded domain in $\mathbb{R}^{n}, n \geq 2$, with $0 \in \Omega$. For any $1<p<n$, the well-known Hardy-Sobolev inequality

$$
\int_{\Omega}|\nabla u|^{p} d x \geq\left(\frac{n-p}{p}\right)^{p} \int_{\Omega} \frac{|u(x)|^{p}}{|x|^{p}} d x
$$

holds for $u \in W_{0}^{1, p}(\Omega)$, where $W_{0}^{1, p}(\Omega)$ is the completion of $C_{0}^{\infty}(\Omega)$ in the norm $\|u\|_{1, p, \Omega}:=\left(\int_{\Omega}|u(x)|^{p} d x+\int_{\Omega}|\nabla u|^{p} d x\right)^{1 / p}$. It is known that there is no function $u \in W_{0}^{1, p}(\Omega)$ for which the best constant $\beta_{n, p}:=\left(\frac{n-p}{p}\right)^{p}$ is achieved. So, one anticipates to have an estimate of the error term on the right-hand side of the inequality (1.1). For the case $p=2$, such improved Hardy-Sobolev inequalities are known. For example, Brezis-Vazquez $[\mathrm{BV}]$ have proved that there exists a constant $C>0$ depending only on $n, q$ and $\Omega$ such that

$$
\int_{\Omega}|\nabla u|^{2} d x \geq\left(\frac{n-2}{2}\right)^{2} \int_{\Omega} \frac{|u(x)|^{2}}{|x|^{2}} d x+C\left(\int_{\Omega}|u(x)|^{q} d x\right)^{2 / q}
$$

Received by the editors July 5,2000 .

1991 Mathematics Subject Classification. Primary 35J30.

Key words and phrases. Hardy-Sobolev inequality, eigenvalue, p-laplacian.

The second author was supported in part by CSIR, India.

The third author acknowledges funding from the Indo-French Center for Promotion of Advanced Research, under project 1901-02. 
holds for any $u \in H_{0}^{1}(\Omega)$ and $1<q<\frac{2 n}{n-2}$. Chaudhuri and Ramaswamy [CR] have improved the above inequality by introducing a weight function $f(x)=|x|^{-\beta}$ for $0 \leq \beta<2$ :

$$
\int_{\Omega}|\nabla u|^{2} d x \geq\left(\frac{n-2}{2}\right)^{2} \int_{\Omega} \frac{|u(x)|^{2}}{|x|^{2}} d x+C\left(\int_{\Omega} \frac{|u(x)|^{q}}{|x|^{\beta}} d x\right)^{2 / q}
$$

for any $u \in H_{0}^{1}(\Omega)$ and $1<q<2_{\beta}^{*}:=\frac{2(n-\beta)}{n-2}$. Recently, Cabre and Martel have considered critical potentials in their study of existence versus instantaneous explosion for the heat equation with singular potentials CM2], where they have an improved inequality similar to (1.4) below for the case $n=2=p$.

Our aim in this article is to achieve an "optimal" improvement of the inequality (1.1) by adding a second term involving the singular weight $\left(\frac{1}{\log (1 /|x|}\right)^{2}$, in the sense that the improved inequality holds for this weight but fails for any weight more singular than this one. The improvement of inequality (1.1) for the case $1<p<2$ is more delicate than for the case $p \geq 2$. As far as our understanding goes, it is because of the fact that for $1<p<2,(1+x)^{p}, x \geq-1$, does not have a global estimate in terms of either $x^{2}$ or $|x|^{p}$.

Finally, we use our improved inequality to determine exactly when the first eigenvalue of the weighted eigenvalue problem for the operator

$$
L_{\mu} u:=-\left(\operatorname{div}\left(|\nabla u|^{p-2} \nabla u\right)+\frac{\mu}{|x|^{p}}|u|^{p-2} u\right)
$$

will tend to 0 as $\mu$ increases to $\beta_{n, p}$. Note that the operator $L_{\mu}$ is a positive operator for $0<\mu \leq \beta_{n, p}$, thanks to the Hardy-Sobolev inequality, and is unbounded from below for $\mu>\beta_{n, p}$ (see [GP], Lemma 3.1). Before stating our main results let us introduce the following notations: $\log ^{(1)}():.=\log ($.$) and \log ^{(k)}():.=\log \left(\log ^{k-1}().\right)$ for $k \geq 2$. Our main results are

Theorem 1.1. Let $R \geq \sup _{\Omega}\left(|x| e^{2 / p}\right)$ and $1<p \leq n$. Then there exists $C>0$, depending on $n, p$ and $R$ such that

$$
\int_{\Omega}|\nabla u|^{p} d x \geq\left(\frac{n-p}{p}\right)^{p} \int_{\Omega} \frac{|u(x)|^{p}}{|x|^{p}} d x+C \int_{\Omega} \frac{|u(x)|^{p}}{|x|^{p}}\left(\log \frac{R}{|x|}\right)^{-\gamma} d x
$$

for any $u \in W_{0}^{1, p}(\Omega)$ if and only if

(i) $\gamma \geq 2$ when $1<p<n$,

(ii) $\gamma \geq n$ when $p=n$.

More generally, for $2 \leq p<n$ and for any $1<q<p_{\beta}^{*}:=\frac{p(n-\beta)}{n-p}, 0 \leq \beta<p$, there exists $C_{1}>0$ depending on $n, p, q, R$ and $\Omega$ such that, for any $u \in W_{0}^{1, p}(\Omega)$,

$$
\begin{aligned}
\int_{\Omega}|\nabla u|^{p} d x & \geq\left(\frac{n-p}{p}\right)^{p} \int_{\Omega} \frac{|u(x)|^{p}}{|x|^{p}} d x+C \int_{\Omega} \frac{|u(x)|^{p}}{|x|^{p}}\left(\log \frac{R}{|x|}\right)^{-2} d x \\
& +C_{1}\left(\int_{\Omega} \frac{|u(x)|^{q}}{|x|^{\beta}} d x\right)^{p / q} .
\end{aligned}
$$


One can show using this theorem

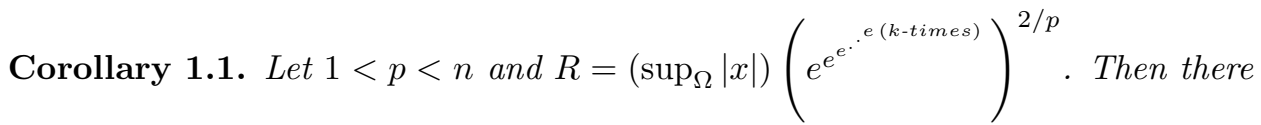
exists $C>0$ depending on $n, p$ and $R$ such that

$$
\int_{\Omega}|\nabla u|^{p} d x \geq\left(\frac{n-p}{p}\right)^{p} \int_{\Omega} \frac{|u(x)|^{p}}{|x|^{p}} d x+C \sum_{j=1}^{k} \int_{\Omega} \frac{|u(x)|^{p}}{|x|^{p}}\left(\prod_{i=1}^{j} \log ^{(i)} \frac{R}{|x|}\right)^{-2} d x
$$

for any $u \in W_{0}^{1, p}(\Omega)$.

Remark 1.1. One of the open problems mentioned in BV] (Problem 2) is whether the two terms on the RHS of inequality (1.2) for $q=2$ are just the first two terms of a series or not. Corollary 1.1 for the particular case $p=2$ shows that indeed the series continues until a certain $k$-th term, which depends only on the choice of $R$.

Remark 1.2. Adimurthi and Sandeep in AS have shown that the best constant for the $n$-dimensional Hardy inequality (inequality (1.4), for the case $p=n$ ) is $\left(\frac{n-1}{n}\right)^{n}$. As a consequence of this, the best constant in inequality (1.4) for the case $p=2$ is $\frac{1}{4}$.

Corollary 1.2. Let $1<p<n$ and let

$F_{p}:=\left\{f: \Omega \rightarrow \mathbb{R}^{+} \mid f \in L_{\mathrm{loc}}^{\infty}(\Omega \backslash\{0\})\right.$ with $\left.\limsup _{|x| \rightarrow 0}|x|^{p} f(x)\left(\log \frac{1}{|x|}\right)^{2}<\infty\right\}$.

If $f \in F_{p}$, then there exists $\lambda(f)>0$ such that for all $u \in W_{0}^{1, p}(\Omega)$

$$
\int_{\Omega}|\nabla u|^{p} d x \geq\left(\frac{n-p}{p}\right)^{p} \int_{\Omega} \frac{|u(x)|^{p}}{|x|^{p}} d x+\lambda(f) \int_{\Omega}|u(x)|^{p} f(x) d x .
$$

If $f \notin F_{p}$ and if $|x|^{p} f(x)\left(\log \frac{1}{|x|}\right)^{2}$ tends to $\infty$ as $|x| \rightarrow 0$, then no inequality of type (1.6) can hold.

Consider the weighted eigenvalue problem with a singular weight,

$$
\begin{aligned}
-\left(\operatorname{div}\left(|\nabla u|^{p-2} \nabla u\right)+\frac{\mu}{|x|^{p}}|u|^{p-2} u\right) & =\lambda|u|^{p-2} u f & & \text { in } \Omega, \\
u & =0 & & \text { on } \partial \Omega,
\end{aligned}
$$

where $f \in \Im_{p}$,

$$
\Im_{p}:=\left\{f:\left.\Omega \rightarrow \mathbb{R}^{+}\left|\lim _{|x| \rightarrow 0}\right| x\right|^{p} f(x)=0 \text { with } f \in L_{\mathrm{loc}}^{\infty}(\Omega \backslash\{0\})\right\},
$$

$1<p<n, 0 \leq \mu<\beta_{n, p}$, and $\lambda \in \mathbb{R}$. We look for a weak solution $u \in W_{0}^{1, p}(\Omega)$ of this problem and study the asymptotic behaviour of the first eigenvalues for different singular weights as $\mu$ increases to $\beta_{n, p}$, after which the operator $L_{\mu}$ is no more bounded from below. 
Theorem 1.2. The above problem admits a positive weak solution $u \in W_{0}^{1, p}(\Omega)$ for all $1<p<n$, corresponding to the first eigenvalue $\lambda=\lambda_{\mu}^{1}(f)>0$. Moreover, as $\mu$ increases to $\beta_{n, p}, \lambda_{\mu}^{1}(f) \rightarrow \lambda(f) \geq 0$ for all $f \in \Im_{p}$ and the limit $\lambda(f)>0$ if $f \in F_{p}$. If $f \notin F_{p}$ and if $|x|^{p} f(x)\left(\log \frac{1}{|x|}\right)^{2}$ tends to $\infty$ as $|x| \rightarrow 0$, then the limit $\lambda(f)=0$.

For the case $p=2$, problem (1.7) has been studied with singular weights $f$ like $\left(1 /|x|^{\beta}\right), 0<\beta<2$, in [CR] and for the radial problem with $f=1$ in [CM1.

\section{Proof of Theorem 1.1}

For the proof of the theorem we will need the following lemmas. Lemma 2.2 is an improved 2-dimensional Hardy inequality and a similar one for the case $k=1$ appears in [BrM] (inequality (A.4)) Lemma 2.3 is an $n$-dimensional Hardy inequality. The proof of Lemma 2.1 is deferred to the appendix.

Lemma 2.1. If $p \geq 2$, then there exist positive constants $B$ and $C$ such that

$$
(1+x)^{p} \geq 1+p x+C x^{2}+B|x|^{p} \quad \forall x \geq-1 .
$$

If $1<p<2$ and $M \geq 1$,

$$
(1+x)^{p} \geq \begin{cases}1+p x+2^{p-4} p(p-1) M^{p-2} x^{2}, & \text { for }-1 \leq x \leq M, \\ 1+p x+2^{p-3} p(p-1) x^{p}, & \text { for } x \geq M\end{cases}
$$

Lemma 2.2 (2-dimensional Hardy inequality). For any $h \in C^{1}[a, T], 0 \leq a<T$ and for $R>T$, the following inequality holds for $k=k(R)$ :

$$
\begin{aligned}
\int_{a}^{T}\left|h^{\prime}(r)\right|^{2} r d r & \geq \frac{1}{4} \sum_{j=1}^{k} \int_{a}^{T} \frac{|h(r)|^{2}}{\left(r \prod_{i=1}^{j} \log ^{(i)} R / r\right)^{2}} r d r \\
& +\frac{1}{2} \sum_{j=1}^{k}\left\{h^{2}(a)\left(\prod_{i=1}^{j} \log ^{(i)} R / a\right)^{-1}-h^{2}(T)\left(\prod_{i=1}^{j} \log ^{(i)} R / T\right)^{-1}\right\}
\end{aligned}
$$

where $k(R)$ is the first positive integer for which $0<\log ^{(k)} R / T \leq 1$.

Proof. Let $h$ be in $C^{1}[a, T]$. For $r \in[a, T]$, define $\psi_{1}(r):=(\log R / r)^{-1 / 2} h(r)$. Then

$$
\begin{aligned}
\int_{a}^{T}\left|h^{\prime}(r)\right|^{2} r d r & =\frac{1}{4} \int_{a}^{T} \frac{\left|\psi_{1}(r)\right|^{2}}{(r \log R / r)} d r+\int_{a}^{T}\left|\psi_{1}^{\prime}(r)\right|^{2}(\log R / r) r d r \\
& -\int_{a}^{T} \psi_{1}(r) \psi_{1}^{\prime}(r) d r \\
& =\frac{1}{4} \int_{a}^{T} \frac{|h(r)|^{2}}{(r \log R / r)^{2}} r d r+\int_{a}^{T}\left|\psi_{1}^{\prime}(r)\right|^{2}(\log R / r) r d r \\
& +\frac{1}{2}\left\{\psi_{1}^{2}(a)-\psi_{1}^{2}(T)\right\} .
\end{aligned}
$$

To estimate the middle term on the RHS, we introduce the iterative function

$$
\psi_{2}(r):=\psi_{1}(r)\left(\log ^{(2)} R / r\right)^{-1 / 2} \text {. }
$$


A simple calculation shows that

$$
\begin{aligned}
\int_{a}^{T}\left|h^{\prime}(r)\right|^{2} r d r & =\frac{1}{4} \sum_{j=1}^{2} \int_{a}^{T} \frac{|h(r)|^{2}}{\left(r \prod_{i=1}^{j} \log ^{(i)} R / r\right)^{2}} r d r \\
& +\int_{a}^{T}\left|\psi_{2}^{\prime}(r)\right|^{2}(\log R / r)(\log \log R / r) r d r \\
& +\frac{1}{2} \sum_{j=1}^{2}\left\{h^{2}(a)\left(\prod_{i=1}^{j} \log ^{(i)} R / a\right)^{-1}-h^{2}(T)\left(\prod_{i=1}^{j} \log ^{(i)} R / T\right)^{-1}\right\} .
\end{aligned}
$$

We can again estimate the middle term on the RHS by introducing

$$
\psi_{3}(r):=\psi_{2}(r)\left(\log ^{(3)} R / r\right)^{-1 / 2}
$$

This process can be continued until we reach $k=k(R)$ for which $0<\log ^{(k)} R / T \leq 1$ and by defining $\psi_{k}(r):=\psi_{k-1}(r)\left(\log ^{(k)} R / r\right)^{-1 / 2}$, ignoring the integral involving the term $\left|\psi_{k}^{\prime}(r)\right|^{2}$ we obtain the inequality (2.3).

By the standard approximation argument we have the following corollary.

Corollary 2.1. Given any $T>0$ and for $R>T$, the inequality

$$
\int_{0}^{T}\left|h^{\prime}(r)\right|^{2} r d r \geq \frac{1}{4} \sum_{j=1}^{k} \int_{0}^{T} \frac{|h(r)|^{2}}{\left(r \prod_{i=i}^{j} \log ^{(i)} R / r\right)^{2}} r d r
$$

holds for any radial function $h$ in $H_{0}^{1}(B)$, where $B$ is the ball of radius $T$ centered at origin, and where $k=k(R)$ is the first integer for which $0<\log ^{(k)} R / T \leq 1$.

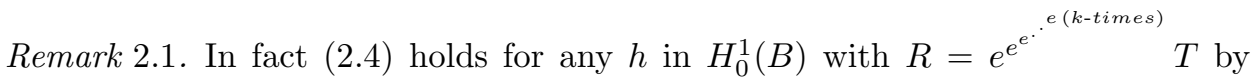
using a symmetrization argument as in the proof of Theorem 1.1 below. This choice of $R$ enables one to conclude that the singular weights $\left(r \prod_{i=1}^{j} \log ^{(i)} R / r\right)^{-2}$ are decreasing functions of $r$ for $1 \leq j \leq k$.

Lemma 2.3 ( $n$-dimensional Hardy inequality). Let $n \geq 2, \Omega=B(0, T) \subset \mathbb{R}^{n}$, be the open ball of radius $T$, centered at origin and $R>T$. Then

$$
\int_{\Omega}|\nabla h|^{n} d x \geq\left(\frac{n-1}{n}\right)^{n} \int_{\Omega} \frac{|h|^{n}}{|x|^{n}}(\log R /|x|)^{-n} d x
$$

for all radial $h \in W_{0}^{1, n}(\Omega)$. 
Proof. For any $h \in C_{0}^{1}(\Omega), h>0$, radially nonincreasing, we define $\psi(r):=$ $(\log R / r)^{-(n-1) / n} h(r)$, for $r \in[0, T]$. Then

$$
\begin{aligned}
h^{\prime}(r) & =\psi^{\prime}(r)(\log R / r)^{(n-1) / n}-\frac{n-1}{n r} \psi(r)(\log R / r)^{-1 / n} \\
& =-\frac{n-1}{n r} \psi(r)(\log R / r)^{-1 / n}\left(1-\frac{n r \psi^{\prime}(r)}{(n-1) \psi(r)} \log R / r\right) .
\end{aligned}
$$

Since $h^{\prime}(r) \leq 0$, we have $\left(1-\frac{n r \psi^{\prime}(r)}{(n-1) \psi(r)}\right) \geq 0$ and hence

$$
\begin{aligned}
\left|h^{\prime}(r)\right|^{n} & =\left(\frac{n-1}{n r}\right)^{n} \psi^{n}(r)(\log R / r)^{-1}\left(1-\frac{n r \psi^{\prime}(r)}{(n-1) \psi(r)} \log R / r\right)^{n} \\
& \geq\left(\frac{n-1}{n r}\right)^{n} \psi^{n}(r)(\log R / r)^{-1}\left(1-n \frac{n r \psi^{\prime}(r)}{(n-1) \psi(r)} \log R / r\right) \\
& =\left(\frac{n-1}{n r}\right)^{n} \psi^{n}(r)(\log R / r)^{-1}-n\left(\frac{n-1}{n r}\right)^{n-1} \psi^{\prime}(r) \psi^{n-1}(r) .
\end{aligned}
$$

Since $\psi(r) \rightarrow 0$ as $r \rightarrow 0$ and $\psi(T)=0$, we obtain

$$
\begin{aligned}
\int_{\Omega}|\nabla h|^{n} d x & =\omega_{n} \int_{0}^{T}\left|h^{\prime}(r)\right|^{n} r^{n-1} d r \\
& \geq \omega_{n}\left(\frac{n-1}{n}\right)^{n} \int_{0}^{T}|h(r)|^{n}(\log R / r)^{-n} \frac{d r}{r} \\
& -\omega_{n}\left(\frac{n-1}{n}\right)^{n-1} \int_{0}^{T}\left(\frac{d}{d r} \psi^{n}(r)\right) d r \\
& =\left(\frac{n-1}{n}\right)^{n} \int_{\Omega} \frac{|h(x)|^{n}}{|x|^{n}}(\log R / r)^{-n} d x .
\end{aligned}
$$

For any radial function $h$ in $W_{0}^{1, n}(\Omega)$, approximate $h$ by smooth radial $h_{m}$. Then using strong convergence of $h_{m}$ to $h$ in the gradient norm and also Fatou's lemma, the above inequality holds for all radial $h$ in $W_{0}^{1, n}(\Omega)$.

Now we are in a position to give a proof of Theorem 1.1 and we organize the proof in following manner: First we prove the validity of inequality (1.4) for the cases $2 \leq p<n$ and $1<p<2$ separately and finally we show the optimality of inequality (1.4).

Proof of Theorem 1.1. Let us assume $1<p<n$ and $\gamma \geq 2$. Since the function $\gamma \mapsto(\log R / r)^{-\gamma}$ is monotonically decreasing on $[2, \infty)$, it is enough to prove the inequality (1.4), only for $\gamma=2$. For both cases $p \geq 2$ and $p<2$, we shall first prove the inequalities for smooth positive radially nonincreasing functions defined on a ball $B$, centered at 0 and of radius $T$. For $u \in C_{0}^{2}(B), u>0$, radially nonincreasing, we define

$$
v(r):=u(r) r^{(n-p) / p}, r=|x|
$$


Here without loss of generality we as well assume $u^{\prime}(r)<0$ (replacing $u$ by $u+$ $\epsilon(T-r)$ for $\epsilon>0$, sufficiently small). Now we observe that

$$
\begin{aligned}
& \int_{B}|\nabla u|^{p} d x-\beta_{n, p} \int_{B} \frac{|u(x)|^{p}}{|x|^{p}} d x=\omega_{n} \int_{0}^{T}\left|\frac{n-p}{p} r^{-n / p} v(r)-r^{1-n / p} v^{\prime}(r)\right|^{p} r^{n-1} d r \\
& -\beta_{n, p} \omega_{n} \int_{0}^{T} \frac{v^{p}(r)}{r} d r \\
& =\omega_{n} \beta_{n, p} \int_{0}^{T} v^{p}(r)\left\{\left|1-\frac{p v^{\prime}(r) r}{(n-p) v(r)}\right|^{p}-1\right\} \frac{d r}{r} .
\end{aligned}
$$

Since $u$ is a decreasing function, we have from (2.5) $v^{\prime}(r)-\frac{(n-p) v(r)}{p r}<0$ and we call $x(r):=-\frac{p v^{\prime}(r) r}{(n-p) v(r)}$ so that $x(r)>-1$. Now we consider the following cases:

Case $p \geq 2$ : By inequality (2.1) in Lemma 2.1, and from (2.6), we obtain

$$
\begin{aligned}
& \int_{B}|\nabla u|^{p} d x-\beta_{n, p} \int_{B} \frac{|u(x)|^{p}}{|x|^{p}} d x \\
& \geq C \omega_{n}\left(\frac{n-p}{p}\right)^{p-2} \int_{0}^{T} v^{p-2}(r)\left|v^{\prime}(r)\right|^{2} r d r \\
&+B \omega_{n} \int_{0}^{T}\left|v^{\prime}(r)\right|^{p} r^{p-1} d r-p \omega_{n} \int_{0}^{T} v^{p-1}(r) v^{\prime}(r) d r \\
&= \frac{4 C \omega_{n}}{p^{2}}\left(\frac{n-p}{p}\right)^{p-2} \int_{0}^{T}\left|\left(v^{p / 2}(r)\right)^{\prime}\right|^{2} r d r \\
&+B \omega_{n} \int_{0}^{T}\left|v^{\prime}(r)\right|^{p} r^{p-1} d r,
\end{aligned}
$$

since $v \in C_{0}^{1}(0, T)$. Now by Corollary 2.1,

$$
\begin{aligned}
\int_{0}^{T}\left|\left(v^{p / 2}(r)\right)^{\prime}\right|^{2} r d r & \geq \frac{1}{4} \int_{0}^{T}\left(\frac{v^{p / 2}(r)}{r \log R / r}\right)^{2} r d r \\
& =\frac{1}{4} \int_{0}^{T} \frac{u^{p}(r)}{r^{p}}(\log R / r)^{-2} r^{n-1} d r \\
& =\frac{1}{4} \int_{B} \frac{|u(x)|^{p}}{|x|^{p}}(\log R /|x|)^{-2} d x
\end{aligned}
$$

Now for $0 \leq \beta<p$, we have

$$
\int_{B} \frac{|u(x)|^{q}}{|x|^{\beta}} d x=\omega_{n} \int_{0}^{T}|v(r)|^{q} r^{n-\beta-1-q(n-p) / p} d r .
$$


Notice that for $C^{1}$ radial function $v(r)$ vanishing at $T$,

$$
\begin{aligned}
|v(r)| & =\left|\int_{r}^{T} v^{\prime}(t) d t\right|=\left|\int_{r}^{T}\left(v^{\prime}(t) t^{(p-1) / p}\right) t^{-(p-1) / p} d t\right| \\
& \leq\left(\int_{r}^{T}\left|v^{\prime}(t)\right|^{p} t^{p-1} d t\right)^{1 / p}\left(\log \frac{T}{r}\right)^{(p-1) / p}
\end{aligned}
$$

and hence,

$$
\begin{aligned}
\int_{0}^{T}|v(r)|{ }^{q} r^{\sigma-1} d r & \leq\left(\int_{0}^{T}\left|v^{\prime}(r)\right|^{p} r^{p-1} d r\right)^{q / p} \int_{0}^{T} r^{\sigma-1}\left(\log \frac{T}{r}\right)^{q(p-1) / p} d r \\
& =\left(\int_{0}^{T}\left|v^{\prime}(r)\right|^{p} r^{p-1} d r\right)^{q / p} \int_{0}^{\infty} e^{-\sigma r} r^{q(p-1) / p} d r
\end{aligned}
$$

where $\sigma:=n-\beta-q(n-p) / p$. Now the second integral on the right-hand side is convergent iff $\sigma>0$ i.e., $1<q<\frac{p(n-\beta)}{n-p}$. Call

$$
C_{1}:=B\left(\omega_{n}\right)^{1-p / q}\left(\int_{0}^{\infty} e^{-\sigma r} r^{q(p-1) / p} d r\right)^{-p / q}
$$

Then from these we get $B \omega_{n} \int_{0}^{T}\left|v^{\prime}(r)\right|^{p} r^{p-1} d r \geq C_{1}\left(\int_{B} \frac{|u(x)|^{q}}{|x|^{\beta}} d x\right)^{p / q}$. Combining this with (2.8) and (2.7) we get inequality (1.5) and hence (1.4) for radially decreasing, smooth positive functions. Now by density arguments, inequality (1.5) is valid for any $u \in W_{0}^{1, p}(B), u \geq 0$, and radially nonincreasing. For a general domain $\Omega$, we use symmetrization arguments. Let $B_{T}$ be a ball having the same volume as $\Omega$ with $T=\left(n|\Omega| / \omega_{n}\right)^{1 / n}$, where $\omega_{n}=\left|S^{n-1}\right|$ and let $|u|^{*}$ be the symmetric decreasing rearrangement of the function $|u|$. Now observe that, for any $u \in W_{0}^{1, p}(\Omega),|u|^{*} \in W_{0}^{1, p}\left(B_{T}\right)$ and $|u|^{*}>0$ and radially nonincreasing and hence inequality (1.5) holds for $|u|^{*}$. It is well known that the symmetrization does not change the $L^{p}$ - norm, decreases gradient norm and increases the integrals $\int_{\Omega} \frac{|u(x)|^{p}}{|x|^{p}} d x$ and $\int_{\Omega} \frac{|u(x)|^{p}}{|x|^{p}}\left(\log \frac{R}{|x|}\right)^{-2} d x$, since both the singular weights are decreasing functions of $|x|$ under our assumption on $R$, thanks to Hardy's inequality for rearrangements. Hence (1.5) also holds for any $u \in W_{0}^{1, p}(\Omega)$.

Case $1<p<2$ : Since for the case $1<p<2,(1+x)^{p}$ does not have global estimate in terms of either $x^{2}$ or $|x|^{p}$, we do the following decomposition of the interval $[0, T]$. Take $M \geq \max \left\{1, \frac{1}{(n-p)(\log R / T)}\right\}$ and denote $\alpha:=\frac{n-p}{p}$. Now we define the following sets, $A:=\left\{r \in[0, T]: x(r)=-\frac{p v^{\prime}(r) r}{(n-p) v(r)}<M\right\}, B:=$ $\{r \in[0, T]: x(r)>M\}$ and $\Gamma:=\left\{r \in[0, T]: \frac{v^{\prime}(r)}{v(r)}=-\frac{\alpha M}{r}\right\}$, so that $[0, T]=$ $A \cup B \cup \Gamma$. Now observe that on the set $A, \frac{v^{\prime}(r)}{v(r)}>-\frac{\alpha M}{r}$, i.e., iff $\left(\log v(r) r^{\alpha M}\right)^{\prime}>$ 
0, i.e., iff the function $v r^{\alpha M}$ is monotonically increasing. So, if we define the function $g(r)$ to be $v r^{\alpha M}$ for $0 \leq r \leq T$, then

$$
A=\{r \in[0, T]: g(r) \text { is monotonically increasing }\}
$$

and

$$
B=\{r \in[0, T]: g(r) \quad \text { is monotonically decreasing }\}
$$

We now prove the theorem through following steps:

Step I: Let us assume that $\Gamma=\left\{r \in[0, T]: \frac{u^{\prime}(r)}{u(r)}=-\frac{\alpha(M+1)}{r}\right\}$ is a finite set. Now observe that the critical point of the function $v r^{\alpha M}$ is precisely $\Gamma$ and hence its cardinality will be odd. So let, $\Gamma=\left\{a_{1}, a_{2},,,, a_{2 m+1}\right\}$ with $a_{0}:=0<a_{1}<\ldots a_{2 m+1}<a_{2 m+2}:=T$. Hence $[0, T]$ can be decomposed as $[0, T]=A \cup B \cup \Gamma$, with $A=\bigcup_{i=0}^{m}\left(a_{2 i}, a_{2 i+1}\right)$ and $B=\bigcup_{i=0}^{m}\left(a_{2 i+1}, a_{2 i+2}\right)$. We denote $C_{1}(p):=2^{p-4} p(p-1)$. Hence from (2.6) and by inequality (2.2) in Lemma 2.1 , we get

$$
\begin{aligned}
X(u)=\omega_{n} \beta_{n, p}\left[\int_{A} v^{p}(r)\left\{\left|1-\frac{v^{\prime}(r) r}{\alpha v(r)}\right|^{p}-1\right\} \frac{d r}{r}\right. \\
\left.\quad+\int_{B} v^{p}(r)\left\{\left|1-\frac{v^{\prime}(r) r}{\alpha v(r)}\right|^{p}-1\right\} \frac{d r}{r}\right] \\
\geq \omega_{n} \beta_{n, p}\left[\frac{C_{1}(p) M^{p-2}}{\alpha^{2}} \int_{A} v^{p-2}(r)\left(v^{\prime}(r)\right)^{2} r d r+\frac{2 C_{1}(p)}{\alpha^{p}} \int_{B}\left|v^{\prime}(r)\right|^{p} r^{p-1} d r\right] \\
=\omega_{n}\left[\frac{C_{1}(p)}{(M \alpha)^{2-p}} \sum_{i=0}^{m} \int_{a_{2 i}}^{a_{2 i+1}} v^{p-2}(r)\left(v^{\prime}(r)\right)^{2} r d r\right. \\
=\omega_{n}\left[\frac{4 C_{1}(p)}{p^{2}(M \alpha)^{2-p}} \sum_{i=0}^{m} \int_{a_{2 i}}^{a_{2 i+1}}\left|\left(v^{p / 2}(r)\right)^{\prime}\right|^{2} r d r\right. \\
\left.+2 C_{1}(p) \sum_{i=0}^{m} \int_{a_{2 i+1}}^{a_{2 i+2}}\left|v^{\prime}(r)\right|^{p} r^{p-1} d r\right] .
\end{aligned}
$$

For $r \in[0, T]$, define $w(r):=v^{p}(r)(\log R / r)^{-1}$. Now by Lemma 2.2, we have for $0 \leq i \leq m$

$$
\int_{a_{2 i}}^{a_{2 i+1}}\left|\left(v^{p / 2}(r)\right)^{\prime}\right|^{2} r d r \geq \frac{1}{4} \int_{a_{2 i}}^{a_{2 i+1}} \frac{v^{p}(r)}{(r \log R / r)^{2}} r d r-\frac{1}{2}\left\{w\left(a_{2 i+1}\right)-w\left(a_{2 i}\right)\right\} .
$$


Therefore from (2.9) and (2.10), we obtain

$$
\begin{aligned}
X(u) & \geq \frac{\omega_{n} C_{1}(p)}{p^{2}(M \alpha)^{2-p}}\left[\sum_{i=0}^{m} \int_{a_{2 i}}^{a_{2 i+1}} \frac{v^{p}(r)}{(r \log R / r)^{2}} r d r-2 \sum_{i=0}^{m}\left\{w\left(a_{2 i+1}\right)-w\left(a_{2 i}\right)\right\}\right] \\
& +2 \omega_{n} C_{1}(p) \sum_{i=0}^{m} \int_{a_{2 i+1}}^{a_{2 i+2}}\left|v^{\prime}(r)\right|^{p} r^{p-1} d r \\
& =\frac{\omega_{n} C_{1}(p)}{p^{2}(M \alpha)^{2-p}}\left[\sum_{i=0}^{m} \int_{a_{2 i}}^{a_{2 i+1}} \frac{v^{p}(r)}{(r \log R / r)^{2}} r d r\right. \\
& \left.+2\left\{\sum_{i=0}^{m-1}\left[w\left(a_{2 i+2}\right)-w\left(a_{2 i+1}\right)\right]-w\left(a_{2 m+1}\right)\right\}\right] \\
+ & 2 \omega_{n} C_{1}(p) \sum_{i=0}^{m} \int_{a_{2 i}}^{a_{2 i+2}}\left|v^{\prime}(r)\right|^{p} r^{p-1} d r .
\end{aligned}
$$

Let us observe that

$$
w^{\prime}(r)=p v^{p-1}(r) v^{\prime}(r)(\log R / r)^{-1}+\frac{1}{r} v^{p}(r)(\log R / r)^{-2},
$$

and hence for $0 \leq i \leq m,\left(w\left(a_{2 m+2}\right)=w(T)=0\right)$ we have

$$
\begin{aligned}
w\left(a_{2 i+2}\right)-w\left(a_{2 i+1}\right) & =\int_{a_{2 i+1}}^{a_{2 i+2}} w^{\prime}(r) d r \\
& =\int_{a_{2 i+1}}^{a_{2 i+2}} v^{p}(r)(r \log R / r)^{-2} r d r \\
& +p \int_{a_{2 i+1}}^{a_{2 i+2}} v^{p-1}(r) v^{\prime}(r)(\log R / r)^{-1} d r .
\end{aligned}
$$

Hence from (2.11) and (2.12) we have

$$
\begin{aligned}
& X(u) \geq \frac{\omega_{n} C_{1}(p)}{p^{2}(M \alpha)^{2-p}}\left[\sum_{i=0}^{m} \int_{a_{2 i}}^{a_{2 i+1}} \frac{v^{p}(r)}{(r \log R / r)^{2}} r d r+2 \sum_{i=0}^{m} \int_{a_{2 i+1}}^{a_{2 i+2}} \frac{v^{p}(r)}{(r \log R / r)^{2}} r d r\right] \\
& \quad+2 \omega_{n} C_{1}(p) \sum_{i=0}^{m} \int_{a_{2 i+1}}^{a_{2 i+2}}\left\{\left|v^{\prime}(r)\right|^{p} r^{p-1}+\frac{(M \alpha)^{p-2}}{p} v^{p-1}(r) v^{\prime}(r)(\log R / r)^{-1}\right\} d r \\
& \geq \frac{\omega_{n} C_{1}(p)}{p^{2}(M \alpha)^{2-p}} \int_{0}^{T} \frac{v^{p}(r)}{(r \log R / r)^{2}} r d r \\
& \quad+2 \omega_{n} C_{1}(p) \sum_{i=0}^{m} \int_{a_{2 i+1}}^{a_{2 i+2}}\left\{\left|v^{\prime}(r)\right|^{p} r^{p-1}+\frac{(M \alpha)^{p-2}}{p} v^{p-1}(r) v^{\prime}(r)(\log R / r)^{-1}\right\} d r .
\end{aligned}
$$


Now, since $v^{\prime}(r)<-\frac{\alpha M v(r)}{r}$, on $\left(a_{2 i+1}, a_{2 i+2}\right)$ for each $0 \leq i \leq m$, we have

$$
\begin{aligned}
Y(v) & :=\left|v^{\prime}(r)\right|^{p} r^{p-1}+\frac{(M \alpha)^{p-2}}{p} v^{p-1}(r) v^{\prime}(r)(\log R / r)^{-1} \\
& =\left(-v^{\prime}(r)\right)^{p} r^{p-1}+\frac{(M \alpha)^{p-2}}{p} v^{p-1}(r) v^{\prime}(r)(\log R / r)^{-1} \\
& =\left(-v^{\prime}(r)\right) v^{p-1}(r)\left[\left(\frac{-v^{\prime}(r) r}{v(r)}\right)^{p-1}-\frac{(M \alpha)^{p-2}}{p}(\log R / r)^{-1}\right] \\
& \geq\left(-v^{\prime}(r)\right) v^{p-1}(r)\left[(M \alpha)^{p-1}-\frac{(M \alpha)^{p-2}}{p}(\log R / T)^{-1}\right] \\
& \geq 0, \quad \text { iff }(M \alpha)^{p-1}-\frac{(M \alpha)^{p-2}}{p}(\log R / T)^{-1} \geq 0 .
\end{aligned}
$$

Since $M \geq \frac{1}{n-p}(\log R / T)^{-1}=\frac{1}{p \alpha}(\log R / T)^{-1}, Y(v) \geq 0$ on each of the intervals $\left(a_{2 i+1}, a_{2 i+2}\right), 0 \leq i \leq m$, from (2.13) we get

$$
\begin{aligned}
X(u) & \geq \omega_{n} \frac{(M \alpha)^{p-2} C_{1}(p)}{p^{2}} \int_{0}^{T} \frac{v^{p}(r)}{(r \log R / r)^{2}} r d r \\
& =\frac{(M \alpha)^{p-2} C_{1}(p)}{p^{2}} \int_{B_{T}} \frac{|u(x)|^{p}}{|x|^{p}}\left(\log \frac{R}{|x|}\right)^{-2} d x .
\end{aligned}
$$

Step II : If the set $\Gamma$ is not finite, we proceed as follows. Define the function $\phi(r):=r u^{\prime}(r)+\delta u(r)$ for $r \in[0, T], \delta:=\alpha(M+1)$. Observe that $\phi \in C^{1}[0, T]$, $\phi(0)=\delta u(0)>0, \phi(T)=T u^{\prime}(T)<0$ and $\Gamma=\phi^{-1}\{0\}$. By Sards Theorem we know that regular values of $\phi$ are dense in $\mathbb{R}$, so there exists $\epsilon_{k} \delta>0$, regular values of $\phi$ such that $\epsilon_{k} \rightarrow 0$, as $k \rightarrow \infty$. Thus for $\epsilon_{k}<u(0)$, the set $\phi^{-1}\left\{\epsilon_{k} \delta\right\}=\left\{r \in[0, T]: \frac{\left(u(r)-\epsilon_{k}\right)^{\prime}}{u(r)-\epsilon_{k}}=-\frac{\delta}{r}\right\}$ is finite. Denoting $u_{k}(r):=u(r)-$ $\epsilon_{k}$, then there exists $T_{k}>0$ such that $u_{k}^{\prime}<0$ on $\left[0, T_{k}\right], u_{k}\left(T_{k}\right)=0$ and $T_{k} \rightarrow T$ as $k \rightarrow \infty$. Now by Step I, we have inequality (2.14) for $u_{k}$ on $\left[0, T_{k}\right]$. Since the constant in (2.14) does not depend on $k$, passing through the limit as $k \rightarrow \infty$ we get inequality (2.14) for any smooth, positive and strictly radially decreasing functions in $W_{0}^{1, p}(\Omega)$.

Step III : Now the remaining proof of inequality (1.4) will follow from (2.14) together with the symmetrization arguments as in the case of $p \geq 2$.

Case $p=n$ : In this case inequality (1.4) follows immediately from Lemma 2.3 together with symmetization arguments.

Optimality. Now suppose $1<p<n$ and $0 \leq \gamma<2$. Since $\beta_{n, p}$ is the best constant for inequality (1.1), the theorem follows for the case $\gamma=0$. So we assume $0<\gamma<2$. The theorem will follow if we can prove for the unit ball $B$ that

$$
\inf _{u \in W_{0}^{1, p}(B) \backslash\{0\}} I_{\gamma}(u):=\frac{\int_{B}\left(|\nabla u|^{p}-\beta_{n, p} \frac{|u(x)|^{p}}{|x|^{p}}\right) d x}{\int_{B} \frac{|u(x)|^{p}}{|x|^{p}}(\log R /|x|)^{-\gamma} d x}=0 .
$$


Now, our aim is to construct a family $u_{\epsilon} \in W_{0}^{1, p}(B), \epsilon>0$, such that

$$
\lim _{\epsilon \rightarrow 0} I_{\gamma}\left(u_{\epsilon}\right)=0
$$

and then (2.15) will follow. For $\epsilon>0$ sufficiently small, let us define

$$
u_{\epsilon}(r):= \begin{cases}0, & \text { for } r \leq \epsilon^{2}, \\ \frac{\log r / \epsilon^{2}}{r^{(n-p) / p} \log 1 / \epsilon}, & \text { for } \epsilon^{2} \leq r \leq \epsilon \\ \frac{\log 1 / r}{r^{(n-p) / p} \log 1 / \epsilon}, & \text { for } \epsilon \leq r \leq 1\end{cases}
$$

Clearly, $u_{\epsilon}$ is continuous and differentiable a.e. and its derivative is given by

$$
u_{\epsilon}^{\prime}(r)= \begin{cases}0, & \text { for } 0 \leq r \leq \epsilon^{2}, \\ \frac{1}{r^{n / p} \log 1 / \epsilon}\left[1-\frac{n-p}{p} \log r / \epsilon^{2}\right], & \text { for } \epsilon^{2} \leq r \leq \epsilon, \\ -\frac{1}{r^{n / p} \log 1 / \epsilon}\left[1+\frac{n-p}{p} \log 1 / r\right], & \text { for } \epsilon \leq r \leq 1\end{cases}
$$

Then we have

$$
\begin{aligned}
\int_{B} \frac{\left|u_{\epsilon}(x)\right|^{p}}{|x|^{p}} d x=\frac{\omega_{n}}{(\log 1 / \epsilon)^{p}}\left[\int_{\epsilon^{2}}^{\epsilon}\left(\log r / \epsilon^{2}\right)^{p} \frac{d r}{r}+\int_{\epsilon}^{1}(\log 1 / r)^{p} \frac{d r}{r}\right] \\
=\frac{\omega_{n}}{(p+1)(\log 1 / \epsilon)^{p}}\left[\int_{\epsilon^{2}}^{\epsilon} \frac{d}{d r}\left(\log r / \epsilon^{2}\right)^{p+1} d r-\int_{\epsilon}^{1} \frac{d}{d r}(\log 1 / r)^{p+1} d r\right] \\
=\frac{2 \omega_{n}}{(p+1)}(\log 1 / \epsilon) .
\end{aligned}
$$

Since $\epsilon>0$ is sufficiently small, we have the following estimates, after a change of variables and the use of Neumann series:

$$
\begin{aligned}
\int_{B}\left|\nabla u_{\epsilon}\right|^{p} d x & =\frac{\omega_{n}}{(\log 1 / \epsilon)^{p}}\left[\int_{\epsilon^{2}}^{\epsilon}\left|\alpha \log r / \epsilon^{2}-1\right|^{p} \frac{d r}{r}+\int_{\epsilon}^{1}|1+\alpha \log 1 / r|^{p} \frac{d r}{r}\right] \\
& =\frac{\omega_{n}}{\alpha(\log 1 / \epsilon)^{p}}\left[\int_{0}^{1} r^{p} d r+\int_{0}^{\alpha \log 1 / \epsilon-1} r^{p} d r+\int_{1}^{\alpha \log 1 / \epsilon+1} r^{p} d r\right] \\
& =\frac{\beta_{n, p} \omega_{n}}{(p+1)} \log 1 / \epsilon\left[\left(1-\frac{1}{\alpha \log 1 / \epsilon}\right)^{p+1}+\left(1+\frac{1}{\alpha \log 1 / \epsilon}\right)^{p+1}\right] \\
& =\frac{2 \beta_{n, p} \omega_{n}}{(p+1)} \log 1 / \epsilon+O\left(\frac{1}{\log 1 / \epsilon}\right) .
\end{aligned}
$$

Therefore from (2.17) and (2.18), we conclude that

$$
\int_{B}\left|\nabla u_{\epsilon}\right|^{p} d x-\left(\frac{n-p}{p}\right)^{p} \int_{B} \frac{\left|u_{\epsilon}(x)\right|^{p}}{|x|^{p}} d x=O\left(\frac{1}{\log 1 / \epsilon}\right) .
$$


Finally, for sufficiently small $\epsilon>0$, let us estimate the following integral:

$$
\begin{aligned}
& \int_{B} \frac{\left|u_{\epsilon}(x)\right|^{p}}{|x|^{p}}(\log R /|x|)^{-\gamma} d x \\
&=\frac{\omega_{n}}{(\log 1 / \epsilon)^{p}}\left[\int_{\epsilon^{2}}^{\epsilon} \frac{\left(\log r / \epsilon^{2}\right)^{p}}{r(\log R / r)^{\gamma}} d r+\int_{\epsilon}^{1} \frac{(\log 1 / r)^{p}}{r(\log R / r)^{\gamma}} d r\right] \\
& \geq \frac{\omega_{n}}{(\log 1 / \epsilon)^{p}}\left[\left(\log R / \epsilon^{2}\right)^{-\gamma} \int_{\epsilon^{2}}^{\epsilon}\left(\log r / \epsilon^{2}\right)^{p} \frac{d r}{r}\right. \\
&\left.+(\log R / \epsilon)^{-\gamma} \int_{\epsilon}^{1}(\log 1 / r)^{p} \frac{d r}{r}\right] \\
& \geq \frac{\omega_{n}}{(p+1)} \log 1 / \epsilon\left[\left(\log R / \epsilon^{2}\right)^{-\gamma}+(\log R / \epsilon)^{-\gamma}\right] \\
& \geq \frac{\omega_{n}}{(p+1)} \log 1 / \epsilon\left[\left(2 \log 1 / \epsilon^{2}\right)^{-\gamma}+(\log 1 / \epsilon)^{-\gamma}\right] \\
&= C(\log 1 / \epsilon)^{1-\gamma} .
\end{aligned}
$$

Since $0<\gamma<2, I_{\gamma}\left(u_{\epsilon}\right) \rightarrow 0$, as $\epsilon \rightarrow 0$ and hence the theorem for the case $1<p<n$.

For the case $p=n$, by taking the same test functions $u_{\epsilon}$ with $p=n$, it is easy to verify that $\int_{B}\left|\nabla u_{\epsilon}\right|^{n} d x=\frac{2 \omega_{n}}{(n+1)}(\log 1 / \epsilon)^{1-n}$ and $\int_{B} \frac{\left|u_{\epsilon}(x)\right|^{n}}{|x|^{n}}(\log R /|x|)^{-\gamma} d x \geq$ $C(\log 1 / \epsilon)^{1-\gamma}$ and hence the optimality.

Proof of Corollary 1.1. In the proof of Theorem 1.1, we have used only the first term of the series in 2-dimensional Hardy inequality. By making use of Lemma 2.2 and Corollary 2.1 in its full generality, the Corollary will follow. In particular, in (2.8), we use inequality (2.4) for the case $2 \leq p<n$ and in (2.10), we use inequality (2.3) for the case $1<p<2$. But in this case, the proof is a bit more cumbersome because of the presence of more boundary terms in (2.10), which can be again handled by considering iterative functions similar to the ones defined in the proof of Lemma 2.2 and following the same line of the proof of Theorem 1.1.

Proof of Corollary 1.2. If $f \in F_{p}$, then

$$
\lim _{\epsilon \rightarrow 0} \sup _{x \in B_{\epsilon}} f(x)|x|^{p}\left(\log (1 /|x|)^{2}<\infty\right.
$$

and hence for sufficiently small $\epsilon$, in $B_{\epsilon}$

$$
f(x)<\frac{C}{|x|^{p}(\log 1 /|x|)^{2}} .
$$

Outside $B_{\epsilon}$, both are bounded functions and hence $C$ can be chosen so that this inequality holds in $\Omega$. Then (1.6) will follow from (1.4).

If $f \notin F_{p}$ and if $|x|^{p} f(x)\left(\log \frac{1}{|x|}\right)^{2}$ tends to $\infty$ as $|x| \rightarrow 0$, then we can write $f(x)=h(x) /|x|^{p}(\log 1 /|x|)^{2}$, where $h(x)$ tends to infinity as $x$ tends to 0 . Then 
from the calculations of Theorem 1.1, for $\epsilon>0$ sufficiently small

$$
\begin{aligned}
\int_{B} \frac{\left|u_{\epsilon}(x)\right|^{p} h(x)}{|x|^{p}\left(\log (1 /|x|)^{2}\right)} d x & =\frac{\omega_{n}}{(\log 1 / \epsilon)^{p}}\left[\int_{B_{\epsilon} \backslash B_{\epsilon^{2}}} \frac{\left(\log |x| / \epsilon^{2}\right)^{p} h(x)}{|x|^{n}(\log R /|x|)^{2}} d x\right. \\
& \left.+\int_{B_{1} \backslash B_{\epsilon}} \frac{(\log 1 /|x|)^{p} h(x)}{|x|^{n}(\log R /|x|)^{2}} d x\right] \\
& \geq \frac{\omega_{n} \inf _{B_{\epsilon} \backslash B_{\epsilon^{2}} h(x)}^{(\log 1 / \epsilon)^{p}}\left(\log R / \epsilon^{2}\right)^{-2} \int_{\epsilon^{2}}^{\epsilon}\left(\log r / \epsilon^{2}\right)^{p} \frac{d r}{r}}{} \\
& \geq \frac{\omega_{n} m(\epsilon) \log 1 / \epsilon}{(p+1)\left(\log R / \epsilon^{2}\right)^{2}} \\
& \geq \frac{\omega_{n} m(\epsilon) \log 1 / \epsilon}{(p+1)\left(2 \log 1 / \epsilon^{2}\right)^{2}} \\
& =C \frac{m(\epsilon)}{\log 1 / \epsilon} .
\end{aligned}
$$

Here $m_{\epsilon}$ stands for $\inf _{B_{\epsilon} \backslash B_{\epsilon^{2}}} h(x)$. Since $m_{\epsilon}$ tends to $\infty$ as $\epsilon \rightarrow 0$, we conclude that $I_{f}\left(u_{\epsilon}\right) \rightarrow 0$ as $\epsilon \rightarrow 0$ and inequality (1.6) cannot hold for such $f \notin F_{p}$.

\section{Proof of Theorem 1.2}

In defining the eigenvalues for the operator $\left(\operatorname{div}\left(|\nabla u|^{p-2} \nabla u\right)+\frac{\mu}{|x|^{p}}|u|^{p-2} u\right)$ in a given domain $\Omega \subseteq \mathbb{R}^{n}$, we interpret the first equation in (1.7) in the following weak sense:

$$
\int_{\Omega}|\nabla u|^{p-2}\langle\nabla u, \nabla \phi\rangle d x=\lambda \int_{\Omega}|u|^{p-2} u \phi d x
$$

whenever $\phi \in W_{0}^{1, p}(\Omega)$.

In order to prove the theorem we need the following two lemmas; the first one is due to Boccardo and Murat [BM] and the second one is a standard result from measure theory (see for example, $[\underline{S}]$, Chapter 1, section 4). The use of Lemma 3.1 here is inspired by the arguments in GP.

Lemma 3.1 (see Theorem 2.1 in $[\mathrm{BM}]$ ). Let $\left(u_{m}\right)_{m \in \mathbb{N}} \subset W_{0}^{1, p}(\Omega)$ be such that as $k \rightarrow \infty, u_{m} \rightarrow u$ weakly in $W_{0}^{1, p}(\Omega)$ and satisfies

$$
-\left(\operatorname{div}\left(\left|\nabla u_{m}\right|^{p-2} \nabla u_{m}\right)\right)=f_{m}+g_{m}
$$

in $D^{\prime}(\Omega)$ where $f_{m} \rightarrow 0$ strongly in $W^{-1, p^{\prime}}(\Omega)$ and $g_{m}$ is bounded in $M(\Omega)$, the space of Radon measures, i.e.

$$
\left|\left\langle g_{m}, \phi\right\rangle\right| \leq C_{K}\|\phi\|_{\infty}
$$

for all $\phi \in D(\Omega)$ with $\operatorname{supp}(\phi) \subset K$. Then there exists a subsequence, say $u_{m_{k}}$, such that

$$
u_{m_{k}} \rightarrow u \text { strongly in } W_{0}^{1, q}(\Omega) \quad \forall q<p .
$$

Lemma 3.2. Let $\left(g_{m}\right)_{m \in \mathbb{N}} \subset L^{p}(\Omega), 1 \leq p<\infty$, be such that, as $m \rightarrow \infty$, (i) $g_{m} \rightarrow g$ weakly in $L^{p}(\Omega)$ and (ii) $g_{m}(x) \rightarrow g(x)$ a.e. in $\Omega$. Then

$$
\lim _{m \rightarrow \infty}\left[\left\|g_{m}\right\|_{p}^{p}-\left\|g_{m}-g\right\|_{p}^{p}\right]=\|g\|_{p}^{p}
$$


Proof of Theorem 1.2. We look for the critical points of the functional

$$
J_{\mu}(u):=\int_{\Omega}|\nabla u|^{p}-\mu \int_{\Omega} \frac{|u(x)|^{p}}{|x|^{p}} d x
$$

which is continuous, Gateaux differentiable and coercive on $W_{0}^{1, p}(\Omega)$ thanks to Hardy-Sobolev inequality. We minimize this functional $J_{\mu}$ over the manifold $M=$ $\left\{\left.u \in W_{0}^{1, p}(\Omega)\left|\int_{\Omega}\right| u(x)\right|^{p} f(x) d x=1\right\}$ and let $\lambda_{\mu}^{1}$ be the infimum. It is clear that $\lambda_{\mu}^{1}>0$. By standard arguments, we can choose a special minimizing sequence $\left(u_{m}\right)_{m \in \mathbb{N}} \subset M$ with $J_{\mu}\left(u_{m}\right) \rightarrow \lambda_{\mu}^{1}$ and the component of $D J_{\mu}\left(u_{m}\right)$ restricted to $M$, tends to 0 strongly in $W^{-1, p^{\prime}}(\Omega)$. The coercivity of $J_{\mu}$ implies that $\left(u_{m}\right)_{m \in \mathbb{N}}$ is a bounded sequence and hence we have for a subsequence, as $k \rightarrow \infty$,

$$
\begin{array}{ll}
u_{m_{k}} \rightarrow u & \text { weakly in } W_{0}^{1, p}(\Omega), \\
u_{m_{k}} \rightarrow u & \text { weakly in } L^{p}\left(\Omega,|x|^{-p}\right), \\
u_{m_{k}} \rightarrow u & \text { strongly in } L^{p}(\Omega) .
\end{array}
$$

Since $W_{0}^{1, p}(\Omega)$ is compactly embedded in $L^{p}(\Omega, f(x)$ ) (see Proposition 2.1 in [CR] $)$, it follows that $M$ is weakly closed and hence $u \in M$. Further $u_{m}$ satisfies in $D^{\prime}(\Omega)$

$$
-\left(\operatorname{div}\left(\left|\nabla u_{m}\right|^{p-2} \nabla u_{m}\right)+\frac{\mu}{|x|^{p}}\left|u_{m}\right|^{p-2} u_{m}\right)=\lambda_{m}\left(\left|u_{m}\right|^{p-2} u_{m} f\right)+f_{m}
$$

where $f_{m} \rightarrow 0$ strongly in $W^{-1, p^{\prime}}(\Omega)$ and $\lambda_{m} \rightarrow \lambda$ as $m \rightarrow \infty$. Calling $g_{m}=\frac{\mu}{|x|^{p}}\left|u_{m}\right|^{p-2} u_{m}+\lambda_{m}\left(\left|u_{m}\right|^{p-2} u_{m} f\right)$, one can check that $g_{m}$ is bounded in $M(\Omega)$. Then we can use Lemma 3.1 to conclude a.e. convergence of $\nabla u_{m_{k}}$ to $\nabla u$ in $\Omega$ and then apply Lemma 3.2 to $u_{m_{k}}$ and also to $\nabla u_{m_{k}}$ to obtain

$$
\begin{aligned}
\lambda_{\mu}^{1} & =\left\|\nabla\left(u_{m_{k}}-u\right)\right\|_{p}^{p}-\mu\left\|u_{m_{k}}-u\right\|_{L^{p}\left(\Omega,|x|^{-p}\right)}^{p}+\|\nabla u\|_{p}^{p}-\mu\|u\|_{L^{p}\left(\Omega,|x|^{-p}\right)}^{p}+o(1) \\
& \geq\left(\beta_{n, p}-\mu\right)\left\|u_{m_{k}}-u\right\|_{L^{p}\left(\Omega,|x|^{-p}\right)}^{p}+\lambda_{\mu}^{1}+o(1)
\end{aligned}
$$

where $o(1) \rightarrow 0 \quad$ as $k \rightarrow \infty$. As $\mu<\beta_{n, p}$, we conclude that

$$
\left\|\left(u_{m_{k}}-u\right)\right\|_{L^{p}\left(\Omega,|x|^{-p}\right)}^{p} \rightarrow 0
$$

as $k \rightarrow \infty$ and also $\left\|\nabla\left(u_{m_{k}}-u\right)\right\|_{L^{p}(\Omega)}^{p} \rightarrow 0$ as $k \rightarrow \infty$ and hence we have $J_{\mu}(u)=\lambda_{\mu}^{1}$ and $\lambda=\lambda_{\mu}^{1}$. Since $J_{\mu}(|u|)=J_{\mu}(u)$, we can take $u>0$ in $\Omega$. Then using Lemma 3.1, $u$ is a distribution solution of (1.7) and since $u \in W_{0}^{1, p}(\Omega)$, it is a weak solution to the eigenvalue problem (1.7), corresponding to $\lambda=\lambda_{\mu}^{1}$. Moreover, if $f \in F_{p}$, by Corollary 1.2 we have

$$
\lambda_{\mu}^{1}(f) \rightarrow \lambda(f)=\inf _{u \in W_{0}^{1, p}(\Omega) \backslash\{0\}} \frac{\int_{\Omega}\left(|\nabla u|^{p}-\beta_{n, p} \frac{|u(x)|^{p}}{|x|^{p}}\right) d x}{\int_{\Omega}|u(x)|^{p} f(x) d x}>0 \text { as } \mu \rightarrow \beta_{n, p} .
$$

If $f \notin F_{p}$, then again by Corollary 1.2, inequality of the type (1.5) cannot hold and hence $\lambda(f)=0$. 


\section{Appendix}

Lemma 2.1. If $p \geq 2$, then there exist positive constants $B$ and $C$ such that

$$
(1+x)^{p} \geq 1+p x+C x^{2}+B|x|^{p} \quad \forall x \geq-1 .
$$

If $1<p<2$ and $M \geq 1$,

$$
(1+x)^{p} \geq \begin{cases}1+p x+2^{p-4} p(p-1) M^{p-2} x^{2}, & \text { for }-1 \leq x \leq M, \\ 1+p x+2^{p-3} p(p-1) x^{p}, & \text { for } x \geq M .\end{cases}
$$

Proof. First let us assume $p \geq 2$, for $x \geq-1$, and define $h_{x}(t):=(1+t x)^{p}$, for all $0 \leq t \leq 1$. Then $h \in C^{2}[0,1]$ and hence by Taylors expansion we have

$$
h_{x}(t)=h_{x}(0)+t h_{x}^{\prime}(0)+t^{2} \int_{0}^{1} h_{x}^{\prime \prime}(\theta t)(1-\theta) d \theta
$$

therefore

$$
\begin{aligned}
h_{x}(1) & =1+p x+p(p-1) x^{2} \int_{0}^{1}(1+t x)^{p-2}(1-t) d t \\
& \geq 1+p x+p(p-1) x^{2} \int_{0}^{1}(1-t)^{p-1} d t \\
& =1+p x+(p-1) x^{2} .
\end{aligned}
$$

Let $0<\alpha<1$ and choose $M>1$, such that for $x \geq M, \alpha x^{p} \geq 1+p x+(\alpha / 2) x^{2}$. Then for $-1 \leq x \leq M$

$$
\begin{aligned}
(1+x)^{p} & \geq 1+p x+(p-1) x^{2} \\
& =1+p x+(\alpha / 2) x^{2}+(p-1-\alpha / 2) x^{2} \\
& \geq 1+p x+(\alpha / 2) x^{2}+M^{2-p}(p-1-\alpha / 2)|x|^{p}
\end{aligned}
$$

and for $x \geq M,(1+x)^{p} \geq x^{p}=\alpha x^{p}+(1-\alpha) x^{p} \geq 1+p x+(\alpha / 2) x^{2}+(1-\alpha) x^{p}$. Combining these inequalities by replacing $C=\alpha / 2$ and

$$
B=\min \left\{1-\alpha, M^{2-p}(p-1-\alpha)\right\},
$$

we obtain (4.1)

Let us consider the case $1<p<2$ and take the function $h_{x}$ for $x>-1$, as above. Notice that $1+t x \neq 0$ and hence $h_{x}^{\prime \prime}$ exists and we have

$$
(1+x)^{p}=1+p x+p(p-1) x^{2} \int_{0}^{1}(1+t x)^{p-2}(1-t) d t .
$$

Now for the case $-1<x \leq M$, from (4.4) we get

$$
\begin{aligned}
(1+x)^{p} & \geq 1+p x+p(p-1) x^{2} \int_{0}^{1 / 2}(1+t x)^{p-2}(1-t) d t \\
& \geq 1+p x+p(p-1) x^{2} \int_{0}^{1 / 2}(2 M)^{p-2}(1-t) d t \\
& =1+p x+\frac{p(p-1)}{4}(2 M)^{p-2} x^{2} \\
& =1+p x+2^{p-4} p(p-1) M^{p-2} x^{2} .
\end{aligned}
$$


Since $M \geq 1$, for the case $x \geq M$, we have from (4.4)

$$
\begin{aligned}
(1+x)^{p} & \geq 1+p x+p(p-1) x^{2} \int_{0}^{1}(2 x)^{p-2}(1-t) d t \\
& =1+p x+2^{p-3} p(p-1) x^{p}
\end{aligned}
$$

Observe that for $x=-1$, inequalities (4.5) and (4.6) are trivially true and hence the lemma.

\section{REFERENCES}

[AS] Adimurthi and Sandeep, Existence and nonexistence of eigenvalue of the perturbed HardySobolev operator, To appear in Proc. Royal Soc. Ed. Sec. A.

[BM] L. Boccardo and F. Murat, Almost everywhere convergence of the gradients of solutions to elliptic and parabolic equations, Nonlinear Anal. TMA. 19 (1992), 581-597. MR 93h:35061

[BrM] H. Brézis and M. Marcus, Hardy's inequalities revisited, Ann. Scuola Norm. Sup. Pisa Cl. Sci (4) xxv (1997), 217-237. MR 99m:46075

[BV] H. Brézis and J. L. Vázquez, Blow-up solutions of some nonlinear elliptic problems, Revista Mat. Univ. Complutense Madrid 10 (1997), 443-469. MR 99a:35081

[CM1] X. Cabré and Y. Martel, Weak eigenfunctions for the linearization of extremal elliptic problems, J. Funct. Anal. 156 (1998), 30-56. MR 99e:35058

[CM2] X. Cabré and Y. Martel, Existence versus instantaneous blow-up for the linear heat equation with singular potentials, C. R. Acad. Sci. Paris, Ser. I, Math 329 (1999), 973-978. MR 2000j:35117

[CR] N. Chaudhuri and M. Ramaswamy, Existence of positive solutions of some semilinear elliptic equations with singular coefficients, To appear in Proc. Royal Soc. Ed. Sec. A.

[GP] Garcia Azorero and I. Peral Alonso, Hardy inequalities and some critical elliptic parabolic problems, Jl. Diff. Equns. 144 (1998), 441-476. MR 99f:35099

[S] M. Struwe, Variational methods applied to nonlinear partial differential equations and Hamiltonian systems, Springer, 1996. MR 98f:49002

School of Mathematics, Tata Institute of Fundamental Research, Bangalore CEntre, IISC CAmpus, Bangalore-560012, India

E-mail address: aditi@math.tifrbng.res.in

Department of Mathematics, Indian Institute of Science, Bangalore-560012, India

E-mail address: cnirmal@math.iisc.ernet.in

School of Mathematics, Tata institute of Fundamental Research, Bangalore CENTRE, IISC CAMPUS, BANGALORE-560012, India

E-mail address: mythily@math.tifrbng.res.in 